Témoigner Témoigner. Entre histoire et mémoire

Getuigen Revue pluridisciplinaire de la Fondation Auschwitz

$118 \mid 2014$

Au nom des victimes. Dictature et terreur d'État en Argentine, Chili et Uruguay

\title{
La Noche de los lápices et les victimes-innocentes
}

La construction de récits autour des lycéens disparus

The narratives construction in relation to secondary school pupils: The Noche de los lápices and the innocent victims

Het verhaal van de Noche de los Lápices. De verdwenen schoolkinderen als 'onschuldige slachtoffers'

\section{Sandra Raggio}

Traducteur : Antonia García Castro

\section{(2) OpenEdition}

\section{Journals}

Édition électronique

URL : http://journals.openedition.org/temoigner/943

DOI : 10.4000/temoigner.943

ISSN : 2506-6390

Éditeur :

Éditions du Centre d'études et de documentation Mémoire d'Auschwitz, Éditions Kimé

Édition imprimée

Date de publication : 1 octobre 2014

Pagination : 106-113

ISBN : 978-2-84174-674-3

ISSN : 2037-4183

Référence électronique

Sandra Raggio, "La Noche de los lápices et les victimes-innocentes », Témoigner. Entre histoire et mémoire [En ligne], 118 | 2014, mis en ligne le 01 octobre 2015, consulté le 23 octobre 2020. URL http://journals.openedition.org/temoigner/943 ; DOI : https://doi.org/10.4000/temoigner.943 


\section{ARGENTINE}

\section{La. Noche de los Lápices et les victimes-innocentes}

La construction de récits autour des lycéens disparus

$\rightarrow$ Par Sandra Raggio,
Universidad Nacional

de La Plata

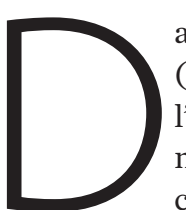

un contexte de défaite militaire, à l'issue de la guerre des Malouine (1982), qui a marqué la fin de la dictature (1976-1983) et le début de ouverture démocratique, la société argentine a été submergée par une masse d'informations à propos des violations des droits de l'homme commises par les militaires. Ce processus de réinformation, selon le

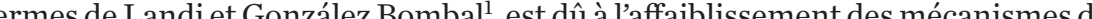

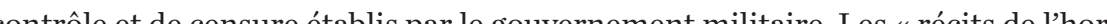

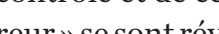
reur» se sont révélés constitutifs de cette nouvelle étape, le principal défiétant alor de légitimer la démocratie naissante par une production de sens en rupture avec le

(1) Inés Gonzalez Bombal et la cultura politica w, in Carlos Acuña et autres, Juicio, castigos
y memoria, Buenos Aires, Nueva
Visión 1995.

(2) Pour plus de précisons, cf.
Inés Gonzźlez Bombal, « Nunca Más. El juicio más allá de los estrados , , in Carlos Acuña et
alli, Juicio, castigos y memorias.
Derechos humanos y justicicie en politica argentina, Buenos Aires, Nueva Visión, 1995 ; Luis Alberto
Romero, «Memorias de El Proceso ", Lucha Armada, $\mathrm{n}^{\circ}$ 2008, Buenos Aires, p. 4-10;
Hugo Vezzetti, Pasado y Presente Guerra, dictaduara y sociedad
en la Argentina, Buenos Aires, Sglo XXI, 2002,

(3) Pour une analyse de lépisode de lhorreur $"$, en référence la couverture médiatique de cadre des premi ères enquêtes sur les disparus, cf. larticle de Cla
Feld dans ce même dossier. passé autoritaire -, la démocratie étant, par définition, tout ce que la dictature avai nié2. L'exhumation des corps de disparus enterrés dans des tombes NN a marqué symboliquement le début des temps nouveaux: la reconstruction de la vérité des faits est apparue commel'un des enjeux les plus importants de la démocratie à venir. Cette sombre image est devenue le symbole de la réémergence de ce qui avait été caché ${ }^{3}$ Sous la dictature, les traces les plus significatives du crime, à savoir les corps et les identités, ontété occultées et les survivants ont été privés de parole, notammen en risondela menacequeles forces armés fat rempre le telle a glenenten patie et elle a également en partie dissipé le soupçon de collaboration avec le régime qui affectait les survivants libérés arbitrairement. Les récits des victimes sont alor devenus des sources dinformation contrant la tentative d'effacement menée par le militaires. De multiples récits de l'horreur ont alors circulé dans les espaces public et privé. Certains sont tombés dans l'oubli, d'autres se sont confinés dans des réseaux de sociabilite restreints ou demeurent dans des mémoires individuelles. En revanche quelques-uns de ces récits ont fait l’objet d'une large diffusion qui s'est répétée. Il est probable que de multiples épisodes resteront encore méconnus, n’ayant pas trouvé de voies de transmission sociale capables de faciliter leur circulation, ou parce qu'ils ont été obstrús par le silence des criminels ou parl'impossibilité de raconter des victimes Ilexisteans victimes. flexiste ainsi un grand nombre de faits dont la trace ne s'est conser

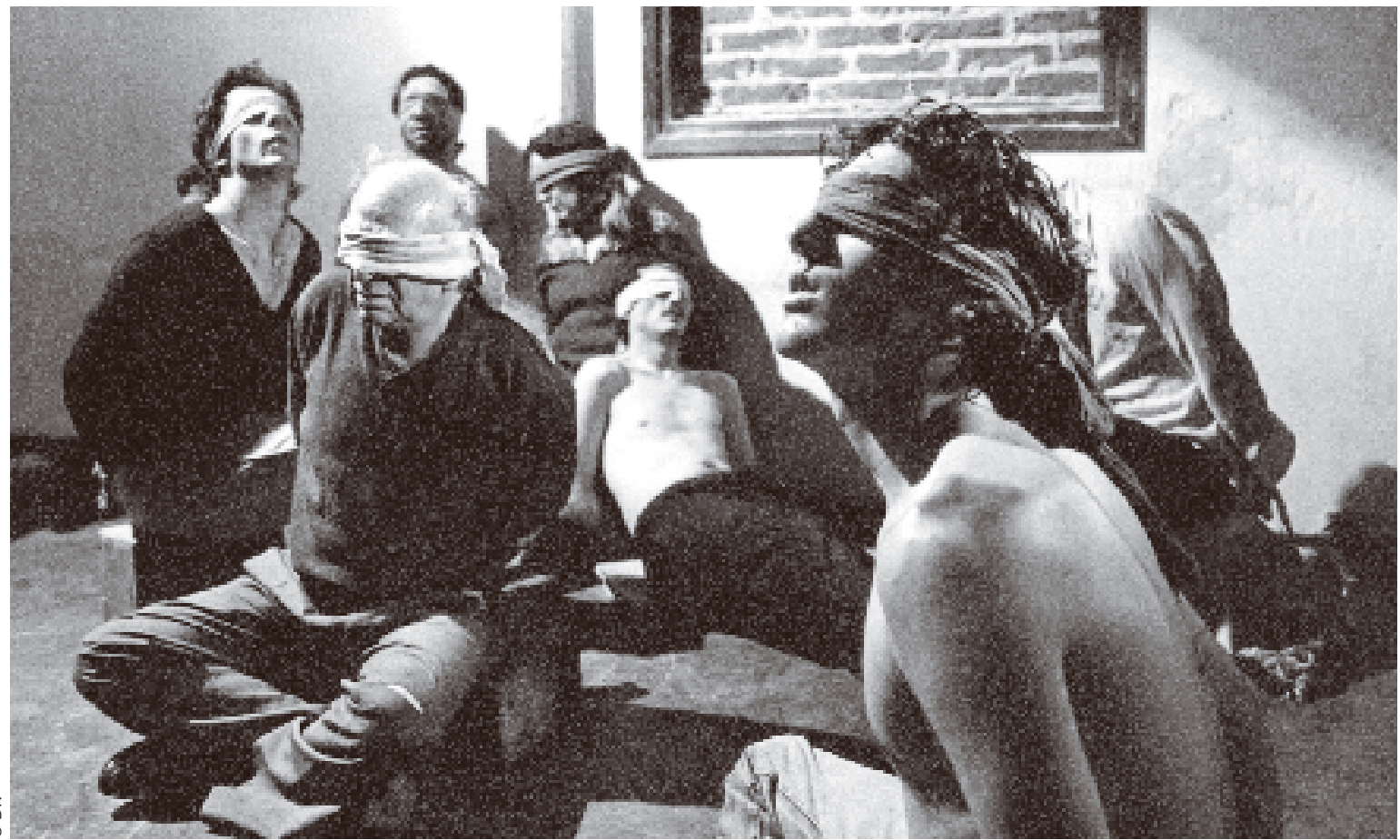

- Image du film La Noche

Néanmoins, chaque fait, connu ou non, formulé ou non, est représenté comme la partie d'un tout, dans le cadre de cette vérité publique construite au cours des premières années de la démocratie qui a nié, de manière radicale, la version racontée par les militaires. Les arguments de la « guerre sale » - selon lesquels la répression s'insérait dans le contexte d’une « guerre antisubversive » marquée par la logique d'exception, cest-à-dire par la criminalisation d'un certain nombre d'actions - ont été confrón aux résultats de l'enqute mén la disparition de personnes (CONADER, 1984). Ces resurs ters figurent dans le rapport Nunca Más (Plus jamais), mettent en relief le fait que les violences perpétrées par le régime ont été menées dans le cadre d'un plan systématique de répression. Ce rapport « exposait une nouvelle vérité publique sur les disparitions et prenait place dans la nouvelle phase interprétative et narrative consistant à juger, penser et évoquer ce passé alors immédiat. ${ }^{4}$ » De même, la sentence prononcée lors du procès des principaux responsables du gouvernement dictatorial en 1985 - le procès des anciens commandants des juntes militaires - allait contester, prenant appui sur des fondements juridiques, l'existence d'une telle guerre et confirmer l'hypothèse d'un plan systématique, établissant ansi une a Vérité indubitable the de

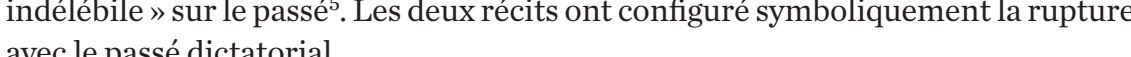

crayons) de Héctor Olivera
(1986) 
Selon Bazcko, « les périodes de crise d’un pouvoir sont aussi celles pendant lesquelles s'intensifie la production d'imaginaires sociaux en compétition ; les représentations d'une nouvelle légitimite et d'un futur prolifèrent et gagnent aussi bien en diffusion qu’en agressivité. ${ }^{6} \gg$ L'utopie démocratique et la mémoire de la dictature se sont bien conjuguées pendant ces premières années. Ce fut l'État qui prit en charge la production de nouvelles sources symboliques de légitimité du pouvoir sur la base d’un imaginaire démocratique qui était à inventer plutôt qưà restaurer «Sur quo

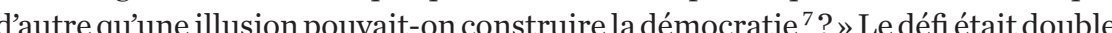
L'État était en concurrence avec, d'une part, le legs de la dictature, d'autre part, des secteurs politiques et sociaux dont les attentes, plus radicales en matière de démocratie, pouvaient se révéler démesurées et renvoyer dangereusement à un passé marqué par l'utopie révolutionnaire et la violence. Le défi était alors de dépasser le passé, non seulement celui de la dictature, mais aussi celui de la conflictualité sociale et politique qui l'avait précédé.

C'est dans ce contexte qu’a été révélé un événement qui par la suite sera très souvent raconté : la Noche de los Lápices (La Nuit des crayons). Il s'agit de l'enlèvement de six adolescents arrêtés dans la nuit du 16 septembre 1976 dans la ville de La Plata, ainsi que de Pablo Diaz le seul qui n’ait pas été tué quelques jours plus tard. Il s’a cissait de lycéens qui avaient milité pour les billets de transport scolire. tarce Raconté de la sorte, le récit a fonctionné depuis les années de transition jusqu’à aujourd'hui comme métonymie de la terreur d'Etat. A maintes reprises, non seulement à l’occasion des anniversaires, la Noche de los Lápices a été évoquée comme exemple condensant «l'Histoire » récente du pays.

La Noche de los Lápices est moins un événement ou une succession d'événements qu'une trame narrative étayée par une série d'épisodes choisis et entrelacé pour construire une interprétation du passé que l'on voulait restituer (une série d'enlèvements survenus dans un laps de temps précis, un groupe de victimes ayant des caractéristiques communes - âge, situation scolaire, lieu de résidence, histoire préalable - et un même mobile). Il s'agit d'une manière de raconter certains faits, réunis sous un nom quiles singularise en tantqu'événement Latrame s'inscrit dans

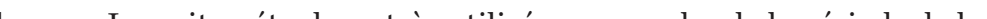
le nom. La nuit, métaphore très utilisée pour parler de la periode de la dictature, renie parleura à (lápices) réfèrent aux protagonistes de cette histoire, les victimes étant des lycéens.

\section{LES VICTIMES-INNOCENTES}

Levénement a eu une résonnance publique à travers le témoignage de Pablo Díaz, présenté comme le «seul survivant » lors du Procès de la junte Militaire, ce qui a motivé la réalisation d'un livre et d'un film homonymes : La Noche de los Lápices. Tous deux ont été amplement diffusés : le livre a été réédité une dizaine de fois et le film mobilise toujours un large public plus de vingt ans après sa sortie. Sa diffusion dans les écoles est une sorte de rituel réitéré chaque 16 septembre. Ce récit coexiste avec d'autres versions de la dictature : celle de la « guerre sale » soutenue par les militaires et la « théorie des deux démons » énoncée par le gouvernement du Parti Radical, au pouvoir depuis la victoire de la combinaison présidentielle « AlfonsínMartinez » aux élections de 1983. Outre le fait que ces récits offraient deux perspectives idéologiques et politiques pour interpréter et juger le passé, ils ont eu un corrélat juridico-pénal. Le propos de « guerre sale » permettait d'exonérer de toute culpabilité tous ceux qui avaient participé à la répression en étant dans l'exercice de leurs fonctions, car ils livraient une juste bataille « contre la subversion ». C'est ce qui explique que ces arguments aentété repris a cor les avocats defenseurs des ce qui explat ces commandants accusés pendant le procès de 1985. Quant à la theorie « des deux démons ", elle responsabilisait les chefs des deux camps, militaires et guérilleros, de a violence utilisée. Le récit de La Noche de los Lápices a eu un impact considérable sur les dimensions pénale et politique de cette théorie que les faits révélés mettaient à mal. Quelle guerre pouvait-on en effet livrer contre des adolescents désarmés qui «ne faisaient que lutter pour un ticket scolaire? » Quels sont les « deux démons » en question?

C'est comme cela que La Noche de los Lápices, dans les versions déjà citées, est devenu un exemple d'un type de récit plus vaste axé sur le «mythe de l'innocence » ou de la « victime innocente ${ }^{8}$ ». Il s'agit d'une manière d'évoquer les personnes disparues pendant la dernière dictature innocentes ici voulant dire non-coupables partes pe dété « enlevées. été enlevées. La caracterstique la plus remarquable du récit est davoir exclu de la narration l'appartenance politique des disparus, notamment à des organisations armées révolutionnaires. Les « hypervictimes », comme les appelle Inés González Bombal $^{10}$, surtout les enfants et les adolescents, ont ici une place prépondérante. Leurs souffrances mettent en évidence et dénoncent le «mal radical », ainsi que le pouvoir fondé sur la disparition ${ }^{11}$

Ces « hypervictimes » ne constituent pas une nouveauté propre à la transition démocratique. Elles avaient déjà une forte présence dans le cadre des dénonciations menées par des organismes des droits de l'homme pendant la dictature. Aussi bien dans le Rapport réclisé par la CIDH (Commission interaméricaine pour les droits pour les droits del'homme delOEA) en 1080 que dans plusieurs documents émanant dorganismes des droits de l'homme, s' institue une catégorie de victimes, celle des disparus adolescents-lycéens, que l'on retrouve ensuite dans la CONADEP, et qui est centrale dans le récit qui nous occupe ici. Ce groupe est, en même temps, un sous-groupe de la catégorie « mineurs ». Dans l'une des publications du CELS (Centre d'études légales et sociales, organisme des droits de l'homme créé à la fin des années 1970) intitulée Adolescents détenus-disparus, on expose avec clarté le type de violence exercée à l'encontre de ce groupe de victimes :
(8) Marcos Novaro et Vicente Dictadura siltiria Argentina 9. L L L $196 / 1993$ Del Golpe de Estado a la restauración democrática, Buenos Aires,
Paidós. 2006 (2é édition). P. 488 .

(9) II convient de signaler que le
Eait que la plupart des disparus ait appartenu à des organisation révolutionnaires ou quils aient
milité au sein d'un autre type d'organisation ne justifie en rien, comme voulait le faire croire Is ont été les victimes et plus ils ont été les victimes et plus
largement les violations des droits
de l'homme qui ont êté commises (10) Gonzalez Bombal, « Nunca Más. El juicio más allá de los (11) Ce récit est tributaire de la innocentes » a été un énoncé soutenu dans des argumentation
juridiques dans la mesure où il juridiques dans la mesure où i
ña jamais êté démontre que ces personnes soient toupabables d'un
poun quelconque délit. Cette manier
de signifier s'est transféré à d'autres récits. Le terme dinnocence a été utilisé comme opposé, lengogetique. Son a été utilisé comment synonyme de de
a résomption de culpabilite. Voir présomption de culpabilité. Voir:
Hugo Vezzetti, Pasado y Presente. Guerra, dictadura y sociedad en la Argentina, Buenos Aires
Siglo XXI, 2002, p. 118 .
Ces jeunes ne se cachaient pas, ils circulaient normalement, ils avaient des relations normales au foyer, au travail ou dans les établissements scolaires qu'ils fréquentaient. Il est ainsi impossible de voir en eux un danger pour la société.

Étant donné également que lors des perquisitions aucune arme n’a été trouvée, pas 
plus qưun quelconque matériel susceptible dêttre considéré comme compromettant, nous sommes en droit de demander : au nom de quelle doctrine, pour conjurer quelle menace, des effectifs armés comme pour un combat, en nombre complètement disproportionné par rapport aux possibilités de résistance, ont été mobilisés contre ces jeunes, qui ne ripostaient pas, et qui ont été traînés menottés et cagoulés, et dans certains cas frappés sans pitié ${ }^{12}$ ?

On a régulièrement cherché à présenter cette victime radicale qui, par la puissance de la révélation de l'horreur qu'elle transmet, devenait un emblème de la tersance de la revélation de l'horreur qu'elle transmet, devenait un emblème de la terreur d'Etat, en omettant tout lien qui pouvait exister entre elle et des activités ou des organisations politiques. Au début de la période démocratique, dans le rapport Nunca Más, la catégorie d’adolescent a pris un caractère central, du fait des caractéristiques conférées à cette catégorie d'âge. « L’adolescent » apparaissait comme un ennemi potentiel de la dictature. Le groupe de «jeunes » concerné par le récit de La Noche de los Lápices est décrit dans les termes suivants :

Ils ne sont pas encore matures, mais ce ne sont plus des enfants. Ils n'ont pas encore pris les décisions fondamentales de la vie, mais ils commencentà tracer leur voie. Its ne pris lesich ne conné achevée lls d'un monde quilis ont herrité de leurs aînés. Chez quelques-uns, un idéal se fait place, un début de rejet devant l'injustice et l'hypocrisie qu'ils combattent parfois de manière auss emphatique que naïve. Cest peut-être parce qu'ils vivent dans leur propre corps des changements vertigineux, qu'ils se méfient de tout ce qui leur est présenté comme immuable ${ }^{13}$.

Ainsi, la tranche d'âge devient un élément clé non seulement pour dénoncer et rejeter la violence déployée par l'État à l'encontre de ces hypervictimes, mais aussi pour l'expliquer en évitant toute considération sur le choix politique des disparus. Dans la préface au rapport Nunca Más, on réaffirme ce type d'aroumentation en soulignant le fait que, dans une grande majorité, les victimes de la tereur d́n soulignant le fait que, dans une terreur d'Etat étaient innocentes pour autant qu'elles netaient pas « coupables de terrorisme ${ }^{14}$ ». Or, dans le contexte de harcèlement et de menace dans lequel ont été menées les actions de dénonciation pendant la dictature, il n'est pas difficile de comprendre le sens stratégique de ces silences. Reste à savoir pourquoi la rupture engendrée par l'instauration de la démocratie n'a pas donné naissance à un autre cadre argumentatif. Elle a bien au contraire renforcé et diffusé les récits existants.

\section{PABLO DIAZ : SURVIVANT, TEMOIN, VICTIME}

Ainsi, en tant que récit, La Noche de los Lápices ajoué un rôle central pour maintenir une certaine manière de rendre compte de la violence répressive. On ne peut expliquer la force de cette histoire sans évoquer la figure de Pablo Díaz qui, à la suite de la parution du livre et du film, a été connu publiquement comme « le seul survi- vant » de ladite « Noche de los Lápices». Nous allons ici analyser son témoignage effectué en 1985 dans le cadre du procès des anciens commandants des juntes militaires. On y retrouve les marques substantielles de cette configuration narrative particulière qui permet de reconstrive la trame du récit dans aspects saillants.

Pablo Díaz a été entendu le 9 mai $1985^{15}$. Le lendemain, tous les journaux ont reproduit des extraits de son témoignage accompagnés de photos du témoin. C'est ainsi qu'un vaste public a lu pour la première fois un récit sur la « Noche de los Lápices » raconté par un

survivan $\mathrm{t}^{16}$. Le Diario del Juicio (Journal du procès) a reproduit les versions sténographiées de trois déclarations liées à cette affaire dont celle de Pablo Díaz Ce jour-lì, ily avatdans la Deve le livre; elle entendait le ténognage pour la première fois. Devant les juges, après avoir répondu aux questions, Pablo Dlaza commencé son témoignage $e^{17}$ en inscrivan son enlèvement dans la saga du 16 septembre (bien qu'il ait été enlevé le 21) et en l'expliquant par la lutte pour le ticket scolaire.

Dr. D’Alessio : Excusez-moi, pour ne pas perdre le fil chronologique, quelle relation avezvous dit que vous aviez avec les autres jeunes gens qui ont été arrêtés ?

Pablo Díaz: Je les ai vus par la suite dans des camps de concentration.

Dr. D'Alessio : Non, je veux dire quels étaient les liens entre vous.

Pablo Díaz : C'était pour le ticket scolaire du secondaire, c’était une demande que nous avions faite, avec la Coorlinadora de Estudiantes Secundarios de La Phata nots plusieurs je plusieurs jeunes tère des Travaux Publics [qui] à ce moment-la s'occupait des transports, nous nous étions rencontrés. Nous nous étions vus mais sans nous connaitre, c'est après, quand je suis tombé sur eux dans différents camps où je me suis retrouvé, que j’ai mis tout cela bout à bout et après les interrogatoires que $j^{\prime}$ ai subis ${ }^{18}$

Pablo Díaz ne faisait pas que raconter ce qu’il avait « vécu », il construisait en même temps une trame en assignant un sens à l'expérience. Les récits qui portent sur la vie ne se confondent pas avec elle, le narrateur a beau avoir vécu ce qu'il raconte, il est toujours un autre, différent de celui qui a vécu les faits. L'expérience racontée tient (n) Si nous confrontons la déclaration de Díaz devant la CONADEP avec celle qưil a
(15) C'est lors de cette audience
que laffaire de « La Noche de los lápices » a été traitée, dautres témoins ont été entendus, mais
aucun de leurs témoignages n'a eul arépercussic
Pablo Díaz. (16) Lui-même fait référence à
cela dans un reportage en 1988
de la revue Nueva Proyección: "'Après, en 84 , jétais au lycée, et des commandants de la junte.
Allors jai fini par âtre le guta personnage du lycée ", Centro de Estudiantes del Colegio Nacional de La Plata, Revista Nueva
Proyección, La Plata, 1988, 33. (17) Diario del Juicio $n^{\circ} 3 \mathrm{du}$

(18) Ibid. p. 62 ; nous soulignons. 
faite au procès, on peut remarquer que cette dernière est plus complète. Entre les deux moments, le récit s'est nourri d'autres déclarations qui ont fourni de nouvelles données, permettant de réélaborer celles dont il disposait. Une voix inconnue prend un nom, une anecdote est associée à une date précise. C'est dans ce sens que l'on peut affirmer que le récit de Pablo Díaz est une fictio ${ }^{19}$, une construction, un «façonnage ». Toutes les déclarations ne sont pas «façonnées » d’une manière si complexe. En général, le premier témoignage est moins narratif, plus proche de la chronique, entre autres, parce qu'il estplus susceptible îtreaffecté par les circonstances dans entre autres Cesquelles il est réalisé.

$\mathrm{Ce}$ «façonnage » comprend une explication des faits vécus. La lutte pour le ticket scolaire est l'antécédent de «l'affaire » instruite dans le cadre du procès. La mise en relation de ces deux événements - la lutte pour le ticket scolaire et son propre enlèvement - ne va pas de soi, elle est la conséquence d'une interprétation de l'expérience. Celle-ci suppose deux opérations. D’un côté, Pablo Díaz reconnaitl'existence de pairs, il peut s'identifier avec eux et préciser ainsi ce qu'il a lui-même vécu. Opérant un rapprochement, il devient « leur » survivant, c'est-à-dire celui qui doit raconter les faits à leur place. C'est pour cela que son récit commence le 16 septembre. Dans le témoignage, «ils» incarnent «l'autre», cet autre qu'il peutévoquer pour se raconte soi-même. Et voilà que le «survivant " la « victime»se transformenten «témoin».

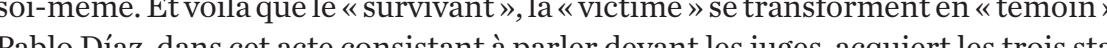
Pablo Diaz, dans cet acte consistant a parler devant les juges, acquiert les trois statuts. Cest ansi quil accomplit mandat du survivant consistant à rendre présents les absents. Pablo Díaz est, de plus, un survivant particulier : il est « unique », non qu'il soit le «seul » témoin, car il y a d’autres survivants pouvant affirmer qu'ils «y étaient » ayant témoigné lors du procès, mais parce qưil se donne cette place suivant le canon interprétatif du discours officiel exprimé dans le rapport Nunca Más. C'est-à-dire qu'il s'institue lui-même comme le « seul mineur-adolescent-lycéen qui a lutté pour le ticket scolaire témoin des faits.»

La production du vraisemblable dans le témoignage du survivant, de celui qui «y était», opère de telle manière que cela dilue la fictio. Le «façonnage» est générépa le récit officiel puisque Pablo Díaz raconte les faits devantle Tribunal commes'illes le récit ócficles avait vecus tel quils sont En même temps, cette composition narrative ancré dans lexpérience traumatique racontée à la première personne confere au témoignage une grande force expressive qui prend encore plus d'importance du fait que l'acte de parole a pour cadre la scène judicaire. Néanmoins, Pablo Díaz ne faisait pas que témoigner devant les juges du tribunal, il racontait aussi son histoire à la société, dans un contexte d'intense déba social et politique sur le sens du passé dans lequel, en dépit de l'intervention de différents acteurs de la vie politique argentine, l'affrontement primordial se jouait entre victimes et bourreaux. L’impact public de son récit montre l'importance qu’a prise cette narration du «mythe de l'innocence » comme moyen de signifier laviolence de la dictature. la dictature. La figure de Pablo Díaz, devenue une icône (la victime par excellence) y a amplement contribué.
L'actualité de ce récit, qui a résisté aux mutations qu’ont connues les narrations sur les disparus et la dictature, nous amène à réfléchir aux continuites qui demeurent dans les modes d'appropriation de l'expérience de la dernière dictature militaire par la société argentine. On pourrait peut-être les expliquer par la présence de certaines strates plus anciennes inscrites dans le sens commun et les imaginaires sociaux cul teciaux qui tend à rieprestion standardises, selon une approche plus universelle qu' historique. Cette observation ouvre certains questionnements autour des limites des explications sur les processus mémoriels qui font appel exclusivement à la dynamique et au contexte politique de production des narrations sur la terreur d'État. Il faudrait alors prêter davantage d'attention à ces codes narratifs qui révèlent, sur le plan symbolique, des dimensions de la vie sociale parfois peu perceptibles. Il faudrait aussi pouvoir examiner les processus d'élaboration sociale du passé sans mettre uniquement l'accent sur les « entrepreneurs » de la mémoire ${ }^{20}$ (les organismes de droits de l'homme, les survivants et les institutions d'État dont l'action impacte les politiques mémorielles), mais en soulignant le rôle d'autres acteurs asecondaires » qui interprètentle passé, (n) généralement en tar que prétendent souvent ces dits «entrepreneurs». 